\title{
Physics-Based Design of Microstrip Magnetic Dipoles Using Cavity Model
}

\author{
Daniel B. Ferreira (1D), Ildefonso Bianchi (1), and Cristiano B. de Paula \\ Department of Microwaves and Optoelectronics - IEEM, Aeronautics Institute of Technology - ITA, \\ Sao Jose dos Campos - SP, Brazil,danielbf@ita.br, ibianchi@ita.br, engcpaula@gmail.com
}

\begin{abstract}
This paper presents an efficient technique of microstrip magnetic dipoles design that explores the physics ruling the antenna behavior not restricting to parametric analyses in a fullwave simulation software. The proposed approach makes use of the cavity model whose parameters are progressively enhanced by using full-wave electromagnetic simulation data in a feedback scheme. A curve fitting problem is established to evaluate the mentioned parameters at each iteration. To exemplify the developed technique, a microstrip magnetic dipole operating at $2.44 \mathrm{GHz}$ (ISM band) was synthesized and its prototype was manufactured and tested in an anechoic chamber. The design was ready in less than one hour and only three full-wave simulations were required. A good agreement between theoretical predictions and experimental results was also observed.
\end{abstract}

Index Terms - Cavity model, microstrip magnetic dipole, modal matching, surrogate-based optimization

\section{INTRODUCTION}

Microstrip magnetic dipoles are a variation of probe-fed rectangular microstrip antennas in which three of the patch edges are shorted to the ground plane by conducting walls. In addition to exhibiting low profile and light weight like microstrip antennas with canonical geometry, they are also compact longitudinally and present low cross-polarization level in $H$-plane [1]. These characteristics make the microstrip magnetic dipoles good candidates for use in printed Yagi antennas and aperiodic linear arrays [2], since such radiators often require both a closer distance between some of their radiating elements and low cross-polarization in both principal planes. In particular, printed Yagi antennas composed of microstrip magnetic dipoles have been extensively discussed in the literature over the last few years [3]-[8] similarly to the design presentation of aperiodic linear arrays of microstrip magnetic dipoles in [9]. Notwithstanding it can operate as a standalone antenna with moderate gain of around $6 \mathrm{dBi}$, rather less attention has been paid to the design of the microstrip magnetic dipole itself, leading to the writing of this paper that presents an efficient technique to assist the microstrip magnetic dipoles design, exploring the physics that rules the antenna behavior.

In this context, the employment of physics-based surrogate models to aid the antenna design is a very advantageous approach [10]. Firstly, because surrogate models are generally non-timeconsuming. Secondly, they can be suitably enhanced from data obtained by a high-fidelity model, which, in general, corresponds to a full-wave electromagnetic simulation with fine discretization 
mesh. Therefore, the use of surrogates provides a highly accurate design that requires only a small number of evaluations of the high-fidelity model. Consequently, the typical design time is shortened. As a comparison, [11] employed a physics-based surrogate consisting of an adapted version of the well-known transmission-line model to perform the design of electrically thick differentially driven probe-fed microstrip antennas. It required only three full-wave simulations to finish the design of the radiator presented in that paper. Reference [12] in turn uses the array factor of an array of isotropic point sources to define a surrogate model that guides the design of linear microstrip antennas arrays. By using this technique, those microstrip arrays spend only three full-wave simulations with fine discretization mesh to be synthesized. Contrastingly, some papers propose the use of artificial neural networks [13] or polynomial regression [14] to assist in the design of printed antennas. But those techniques have the disadvantage of requiring training data points for the building of their models, whose assembly is usually time-consuming. Besides, they often do not explore the physics behind the antenna operation.

In this paper, the cavity model [15] is applied to the microstrip-magnetic-dipole structure and a compact expression to fast compute the antenna input impedance is derived by using the mode matching technique [16]. The same expression is then used as a physics-based surrogate to aid in the tuning of the antenna dimensions throughout the design process. Inherent to the cavity model, the correction factors such as the fringe field length and the feed strip width, which have strong effect on the accuracy of the cavity model, are progressively enhanced with base on the radiator full-wave electromagnetic simulation data obtained in ANSYS HFSS ${ }^{\circledR}$ package. As a consequence, the proposed design technique spends a small number of full-wave simulations to run. Moreover, it allows the understanding of the physics behind the antenna operation in contrast to, e.g., parametric analyses, which are not able to express it for themselves. The developed technique updates the correction factor values through curve fitting that is carried out using a derivative-free routine available in Matlab ${ }^{\circledR}$.

This paper is organized as follows. Section II addresses the microstrip magnetic dipoles design technique, in which all details to formulate the surrogate model are given. Section III reports one application example at $2.44 \mathrm{GHz}$ (ISM band) including experimental results of a prototype with the purpose of verifying the theoretical predictions. Drawn conclusions are depicted in Section IV.

\section{MICROSTRIP MAGNETIC DIPOLE DESIGN TECHNIQUE}

The geometry of a microstrip magnetic dipole in free space (electric permittivity $\varepsilon_{0}$ and magnetic permeability $\left.\mu_{0}\right)$ is depicted in Fig. 1. It consists of a probe-fed rectangular microstrip antenna whose patch has length $a$, width $b$, and is printed on a dielectric substrate of relative permittivity $\varepsilon_{r}$, loss $\operatorname{tangent} \tan \delta$, thickness $h$, length $L$, and width $W$. The rectangular patch is connected to the ground plane through three rows of metallized via holes of diameter $d_{v}$, which are placed close to the back and side edges of the patch and must play the role of conducting walls. The center-to-center spacing between consecutive shorting vias on the back and side edges are equal to $s_{b}$ and $s_{s}$, respectively. In 
addition, the edge of the via holes is one diameter $\left(d_{v}\right)$ apart from the closest edge of the patch and the coaxial probe has a center conductor of radius $r_{f}$ and is positioned at $\left(x_{f}, y_{f}\right)$.

For $d_{v}, s_{b}$, and $s_{s}$ chosen sufficiently small [17], the rows of metallized vias may be modelled by conducting vertical walls located near these rows working as connection between the patch and the ground plane [18]. Hence, if the microstrip magnetic dipole is electrically thin, i.e., $h<<\lambda$ ( $\lambda$ is the wavelength in the dielectric substrate), the cavity model is suitable to evaluate the antenna input impedance $\left(Z_{i n}\right)$ and to estimate the antenna radiation pattern, due to presenting a fine compromise solution between required processing capacity and obtained accuracy. This model allows the designer to quickly assess the influence of each antenna parameter on its $Z_{\text {in }}$ and pattern and does not require a powerful computer to run. Additionally, the model building involves only basic elements of electromagnetic theory, such as resonant cavities and slot radiation.

Cross-sectional views of the equivalent cavity used to model the microstrip magnetic dipole are shown in Fig. 2. This cavity is composed of five electric walls and a magnetic wall located in the plane where $x^{\prime}=a_{e}$ being fed by a coaxial probe. When representing the electric field $(\boldsymbol{E})$ inside the cavity by modal matching [19], i.e., considering that the cavity is formed by two cascaded source-free rectangular waveguide sections of lengths $x_{f}^{\prime}$ and $a_{e}-x_{f}^{\prime}$, height $h$ and width $b_{e}$, and modelling the coaxial probe by a current ribbon centered at $\left(x_{f}^{\prime}, y_{f}^{\prime}\right)$ and with $z^{\prime}$-directed uniform current density yields

$$
\begin{aligned}
& \boldsymbol{E}\left(x^{\prime}, y^{\prime}\right)=\hat{z} \frac{2 \omega \mu_{0}}{j b_{e}} \sum_{n=1}^{\infty} \frac{\sec \left(\beta_{n} a_{e}\right)}{\beta_{n}} \cos \left(\beta_{n}\left(x_{f}^{\prime}-a_{e}\right)\right) \sin \left(\beta_{n} x^{\prime}\right) \sin \left(\frac{n \pi}{b_{e}}\left(y^{\prime}+\frac{b_{e}}{2}\right)\right) J_{f}(n), \text { if } 0 \leq x^{\prime} \leq x_{f}^{\prime} \\
& \boldsymbol{E}\left(x^{\prime}, y^{\prime}\right)=\hat{z} \frac{2 \omega \mu_{0}}{j b_{e}} \sum_{n=1}^{\infty} \frac{\sec \left(\beta_{n} a_{e}\right)}{\beta_{n}} \sin \left(\beta_{n} x_{f}^{\prime}\right) \cos \left(\beta_{n}\left(x^{\prime}-a_{e}\right)\right) \sin \left(\frac{n \pi}{b_{e}}\left(y^{\prime}+\frac{b_{e}}{2}\right)\right) J_{f}(n), \text { if } x_{f}^{\prime} \leq x^{\prime} \leq a_{e}
\end{aligned}
$$

where $\omega$ is the angular frequency, $-b_{e} / 2 \leq y^{\prime} \leq b_{e} / 2, a_{e}$ and $b_{e}$ are the length and width of the equivalent cavity, respectively, $\beta_{n}=\left\{k^{2}-\left(n \pi / b_{e}\right)^{2}\right\}^{1 / 2}$, with $k=k_{0}\left\{\varepsilon_{r}\left(1-j \tan \delta_{e}\right)\right\}^{1 / 2}, k_{0}=\omega\left\{\mu_{0} \varepsilon_{0}\right\}^{1 / 2}, \tan \delta_{e}$ is the effective loss tangent which accounts for the radiated power and losses in the dielectric substrate and metallic walls, and $J_{f}(n)$ is given by [20]

$$
J_{f}(n)=I_{i n} j_{0}\left(\frac{n \pi w_{f}}{2 b_{e}}\right) \sin \left(\frac{n \pi}{b_{e}}\left(y_{f}^{\prime}+\frac{b_{e}}{2}\right)\right), n=1,2, \ldots
$$

that can be interpreted as the Fourier sine transform of the current density on the feed strip whose width is $w_{f}$ and input current is $I_{i n} ; j_{0}(\xi)$ denotes the zero-order spherical Bessel function.

Using the complex Poynting's theorem with the electric field $\boldsymbol{E}$ computed at $x^{\prime}=x_{f}^{\prime}$, the complex power delivered to the equivalent cavity is determined and $Z_{\text {in }}$ is then promptly evaluated as

$$
Z_{\text {in }}=\frac{j 2 \omega \mu_{0} h}{b_{e}} \sum_{n=1}^{\infty}\left\{\frac{\sec \left(\beta_{n} a_{e}\right)}{\beta_{n}} \cos \left(\beta_{n}\left(x_{f}^{\prime}-a_{e}\right)\right) \sin \left(\beta_{n} x_{f}^{\prime}\right) \sin ^{2}\left(\frac{n \pi}{b_{e}}\left(y_{f}^{\prime}+\frac{b_{e}}{2}\right)\right) j_{0}^{2}\left(\frac{n \pi w_{f}}{2 b_{e}}\right)\right\} \text {. }
$$

It is worthwhile to mention that the choice of the mode matching technique to calculate the electric field inside the cavity instead of the expansion in resonant modes [21] - very popular in publications on microstrip antennas - is because the adopted method is less computationally intensive since it 
involves only a single summation to compute the electric field while the discarded method requires the evaluation of a double summation.

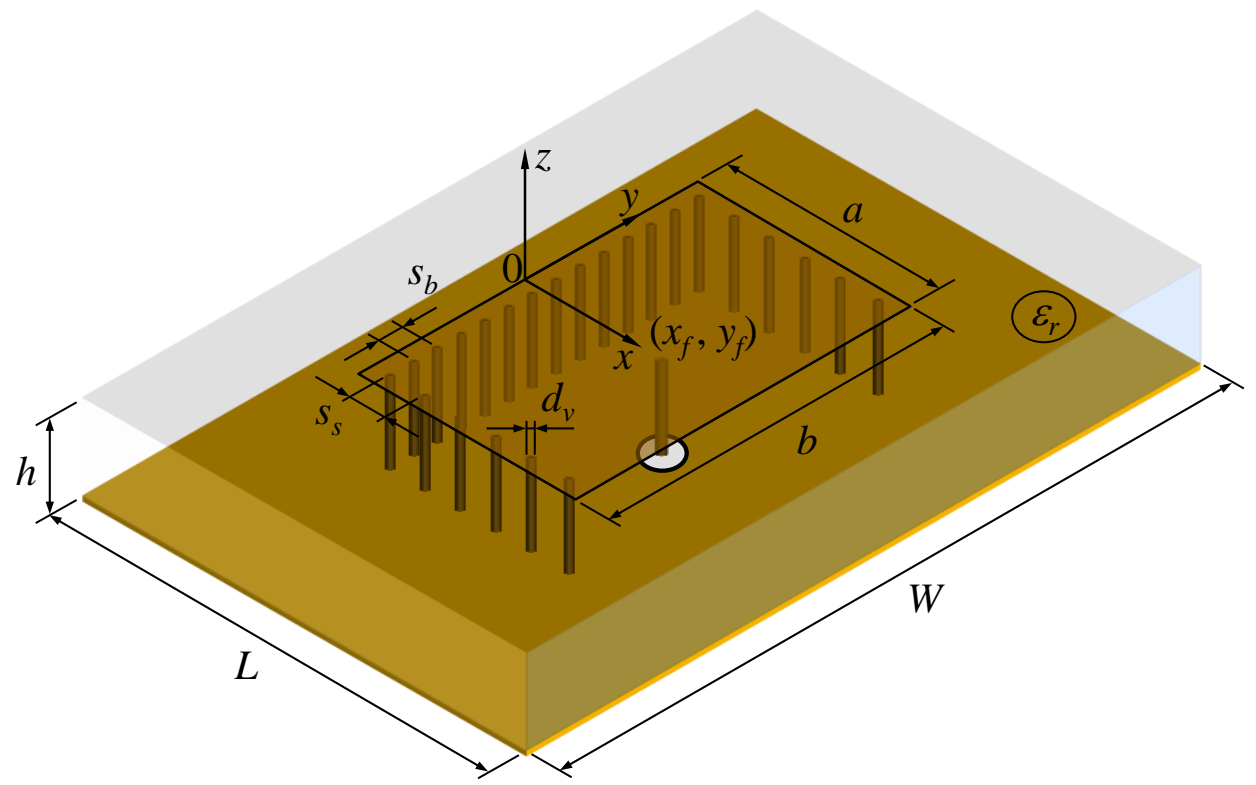

Fig. 1. Microstrip magnetic dipole geometry.

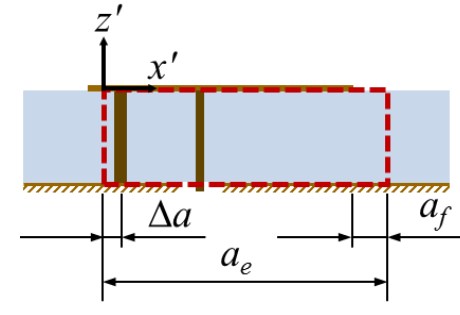

(a) Side view.

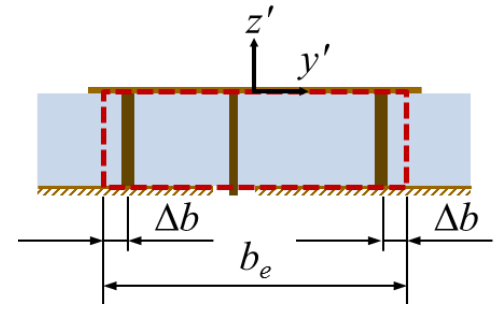

(b) Front view.

Fig. 2. Equivalent cavity dimensions: (a) $x^{\prime} z^{\prime}$-plane; (b) $y^{\prime} z^{\prime}$-plane. Dashed red line indicates the equivalent cavity boundaries.

Based on the picture in Fig. 2 and recalling that the distance between the edge of the via holes and the closest edge of the patch is equal to $d_{v}$, the length $a_{e}$ and width $b_{e}$ of the equivalent cavity may be expressed as

$$
\begin{gathered}
a_{e}=a-1.5 d_{v}-\Delta a+a_{f} \\
b_{e}=b-3 d_{v}-2 \Delta b
\end{gathered}
$$

where $\Delta a$ and $\Delta b$ denote the displacement between the rows of vias and their equivalent conducting walls, and $a_{f}$ accounts for the effect of fringing fields at the front edge of the patch. It was assumed that the position of the equivalent conducting walls is external to the antenna in case of $\Delta a$ or $\Delta b$ negative (case shown in Fig. 2), and internal otherwise. For this reason, $\Delta a$ and $\Delta b$ are subtracted in (5) and (6), respectively. The center coordinates of the current ribbon, in turn, are given by $x_{f}^{\prime}=x_{f}-1.5 d_{v}-\Delta a$ and $y_{f}^{\prime}=y_{f}$. Note that the accuracy of the input impedance evaluated through (4) is highly dependent on the estimates of $\Delta a, \Delta b, a_{f}, w_{f}$, and $\tan \delta_{e}$. Note also that $x^{\prime}=x-a+a_{e}-a_{f}, y^{\prime}=y$, and $z^{\prime}=z$.

As a first approximation, $\Delta a$ and $\Delta b$ are computed from the formulas derived in [18], which establish the equivalence between substrate-integrated waveguides (SIWs) and conventional rectangular waveguides with solid walls, the fringe field extension $a_{f}$ is estimated using the well- 
known expression for microstrip open circuits by Hammerstad [22], and the current ribbon width is approximated by $w_{f} \cong 4 r_{f}$, where $r_{f}$ is the radius of the feed probe. It is still necessary to determine $\tan \delta_{e}$ before calculating $Z_{i n}$. This can be accomplished by following the iterative procedure presented in [19], whose convergence is fast for electrically thin radiators.

The electric field radiated by the microstrip magnetic dipole can be obtained from the equivalent magnetic surface current density $\boldsymbol{M}_{s}$ on the $H$-wall $\left(x^{\prime}=a_{e}\right)$ of the cavity, that is,

$$
\boldsymbol{M}_{s}=-2 \hat{x} \times \boldsymbol{E}\left(a_{e}, y^{\prime}\right)
$$

in which $\boldsymbol{E}\left(a_{e}, y^{\prime}\right)\left(-b_{e} / 2 \leq y^{\prime} \leq b_{e} / 2\right)$ is evaluated from (2). This radiated field is required to compute $\tan \delta_{e}$ in the beginning of the antenna design, as will be explained later.

Usually, the ratio $q$ between width $b$ and length $a$ is set to be greater than or equal to 4 to ensure the compactness of microstrip magnetic dipole along $x$-direction, so that it can be used, e.g., to compose an aperiodic linear array or a printed Yagi antenna as already mentioned. In addition, $a$ and $b$ are dimensioned to have the equivalent cavity operating with one quarter-sine variation in $x$-axis and one half-sine variation in $y$-axis. The coaxial probe is, in turn, positioned on the patch midline, i.e., $y_{f}=0$; consequently, even-order terms of the sums in (1), (2), and (4) vanish and the aforementioned sine variations are excited.

Thus, once $q$ is set, $y_{f}$ is made equal to 0 , and the number of metallized vias on the back and side edges is predefined, $a$ and $x_{f}$ must be tuned to minimize the reflection coefficient magnitude $\left|\Gamma_{i n}\right|$ at the center frequency $f_{0}$ of operating band. This procedure can be carried out through inspection by use of (4), since its computation is quite fast. A suitable initial starting point for this tuning process can be determined assuming one quarter-sine variation in $x$-axis and one half-sine variation in $y$-axis of the equivalent cavity, which leads to approximate values for the wavenumbers $k_{x}$ and $k_{y}$

$$
\begin{aligned}
& k_{x}=\left.\frac{2 \pi}{\lambda_{x}}\right|_{\lambda_{x} \cong 4 a} \cong \frac{\pi}{2 a} \\
& k_{y}=\left.\frac{2 \pi}{\lambda_{y}}\right|_{\lambda_{y} \cong 2 q a} \cong \frac{\pi}{q a} .
\end{aligned}
$$

Additionally, $k_{x}$ and $k_{y}$ are subject to the identity [23]

$$
k_{x}^{2}+k_{y}^{2}=\varepsilon_{r} k_{0}^{2}
$$

in which $k_{0}=\omega\left\{\mu_{0} \varepsilon_{0}\right\}^{1 / 2}$. Thus, for $q=4$ we have

$$
a_{\text {start }}=\left.\frac{\sqrt{q^{2}+4}}{4 q} \frac{c_{0}}{\sqrt{\varepsilon_{r}} f_{0}}\right|_{q=4}=0.28 \frac{c_{0}}{\sqrt{\varepsilon_{r}} f_{0}}
$$

being $a_{\text {start }}$ the initial value for $a$ and $c_{0}$ the speed of light in free space.

Considering that the antenna has also to be matched to $Z_{0}=50 \Omega$, the feed probe can be initially positioned at

$$
x_{f \text { start }}=a_{\text {start }} / 4 \text {. }
$$

For this proposed antenna configuration, (4) shows that to increase the real part of $Z_{i n}$, one must 
move the probe towards the front edge, whereas the plot of $\left|\Gamma_{i n}\right|$ versus frequency shifts to the left as $a$ raises, and vice versa. Addressing these remarks, the tuning process is completed quickly.

However, checking the behavior of the antenna designed according to the previous procedure either by full-wave simulation software like Ansys $\mathrm{HFSS}^{\circledR}$ or by building and testing its prototype, there will likely be a frequency shift in its $\left|\Gamma_{i n}\right|$ (and $Z_{i n}$ ) curve as well as degradation in its impedance matching. This is mainly due to the degree of accuracy of the correction factors $\Delta a, \Delta b, a_{f}, w_{f}$, and $\tan \delta_{e}$, and also due to the effect of dielectric and ground plane truncation that is not accounted for in the cavity model. Therefore, the antenna dimensions must be optimized to actually meet project specifications. A common way to perform this task is using full-wave electromagnetic solvers in an iterative process. But since full-wave simulations demand high computational effort, it is advantageous to have a design strategy involving a small number of simulations. This can be achieved in a straightforward manner by using (4) in a feedback scheme, hence not restricting itself only to the determination of preliminary antenna dimensions as usual.

The proposed optimization technique is divided into five steps as follows.

Step 1: Antenna Predesign. The microstrip magnetic dipole is designed with the aid of (4), (11), and (12) as described before. At this stage, the correction factors $\Delta a, \Delta b, a_{f}, w_{f}$, and $\tan \delta_{e}$ are calculated using the aforementioned approximate models. The impedance bandwidth $B W$ for $\left|\Gamma_{i n}\right| \leq-10 \mathrm{~dB}$ is also evaluated.

Step 2: Full-wave Simulation (FWS). The antenna with dimensions defined in Step 1 is analyzed in a full-wave electromagnetic simulator. Being the specification of $\left|\Gamma_{i n}\right|$ not met, its input impedance $Z_{\text {ref }}$ (real and imaginary parts) is sampled over the frequency range $F=\left[f_{r}-B W / 2, f_{r}+B W / 2\right]\left(f_{r}\right.$ is the frequency closest to $f_{0}$ for which $\left|\Gamma_{i n}\right|$ reaches its minimum value) in steps of $1 \mathrm{MHz}$. At this point, it is necessary to verify if the main beam of the radiation pattern really occurs at broadside at $f_{r}$, otherwise the number of shorting vias must be increased to prevent power leakage through them and the procedure must be repeated from Step 1. It is worth mentioning that in addition to a minimum number of shorting vias, the patch front edge $(x=a)$ is located on the dielectric midline parallel to $y$-axis to present main beam at broadside.

Step 3: Optimization. In this step, the correction factors are optimized to fit the impedance curve (both real and imaginary parts), computed from (4), to the analogous curve obtained through the fullwave simulation $\left(Z_{r e f}\right)$ in the preceding step. To do this, the squared error magnitude between $Z_{\text {in }}$ and $Z_{r e f}$ is minimized in relation to a vector $\boldsymbol{w}$ which contains the five correction factors, i.e., $\boldsymbol{w}=\left[\begin{array}{lll}\Delta a & a_{f} \Delta b & w_{f} \tan \delta_{e}\end{array}\right]$. Thus

$$
\boldsymbol{w}_{\text {opt }}=\arg \min _{w}\left(\sum_{m=1}^{M}\left|Z_{\text {in }}\left(f_{m}\right)-Z_{r e f}\left(f_{m}\right)\right|^{2}\right)
$$

where $\boldsymbol{w}_{\text {opt }}$ represents the optimal solution and $M$ is the number of frequencies $f_{m}$ sampled over the interval $F$. 
The minimization problem defined in (13) may be solved efficiently using, e.g., pattern search methods. One may find implementations of these optimization methods in commercial off-the-shelf numerical computation tools such as Matlab ${ }^{\circledR}$. Besides, to find a meaningful solution from a physical standpoint, the following constraints must be ensured: if $s_{b}>2 d_{v}$, then $\Delta a<0$, otherwise $\Delta a \geq 0$ [18], $a_{f}>0$, if $s_{s}>2 d_{v}$, then $\Delta b<0$, otherwise $\Delta b \geq 0, w_{f}>0$, and $\tan \delta_{e}>\tan \delta$.

Step 4: Retuning. The new correction factors evaluated in Step 3 are utilized in (4) and both patch length $a$ and probe position $x_{f}$ are retuned to set the minimum value of $\left|\Gamma_{i n}\right|$ at frequency $f_{0}$ and with a level lower than or equal to $-40 \mathrm{~dB}$.

Step 5: Synthesis Check. A new full-wave simulation is then performed using the dimensions $a$ and $x_{f}$ updated in the previous step. The plot of $\left|\Gamma_{i n}\right|$ versus frequency is checked enforcing the return to Step 3 in case of the requirement of $\left|\Gamma_{i n}\right|$ not be met.

This iterative method has shown itself capable to converge rapidly, requiring no more than three full-wave simulations in most cases. Fig. 3 presents a block diagram summarizing the main actions required to perform the proposed iterative method.

It is worthwhile to mention that if the three rows of shorting vias were physically replaced by vertical metallic walls located on the edges of the patch, the resulting microstrip magnetic dipole could be designed using the same technique presented in this Section, by simply zeroing the correction factors $\Delta a$ and $\Delta b$, as well as the diameter $d_{v}\left(\Delta a=\Delta b=d_{v}=0\right)$ used on the theoretical framework developed here. Consequently, the optimization is accelerated. Such antenna configuration can be easily assembled using aluminum sheets and air as dielectric, proving itself to be a low-cost solution.

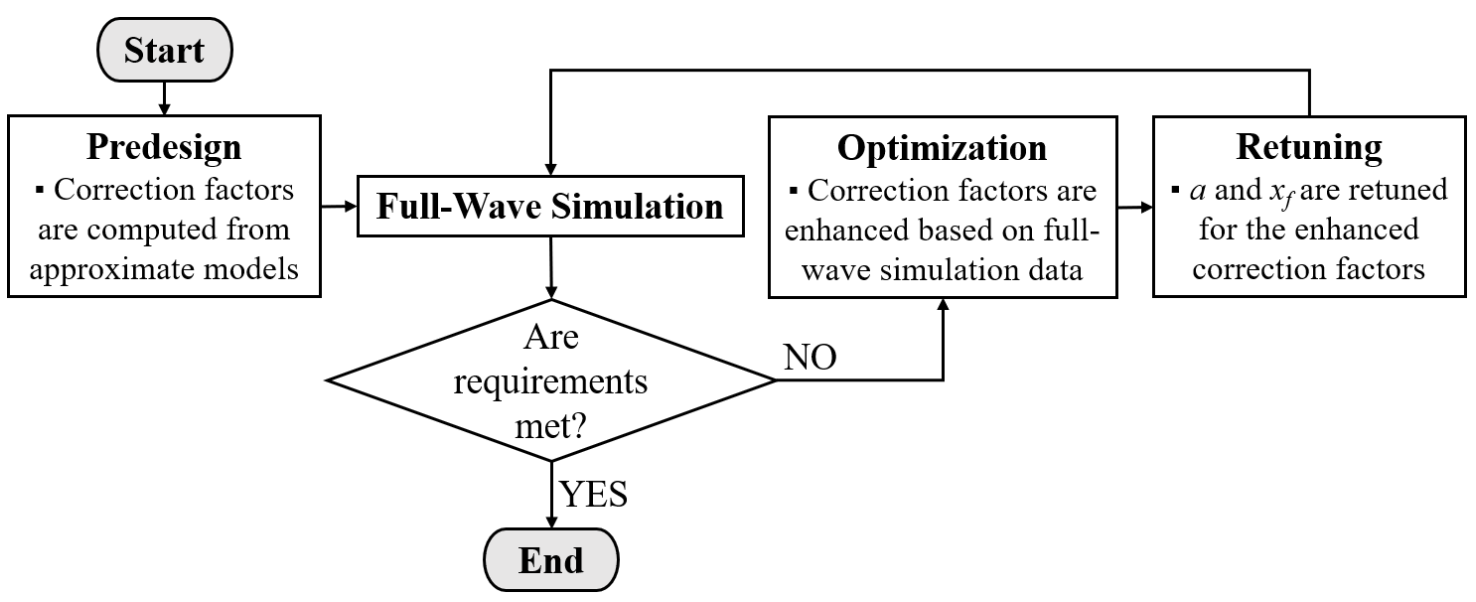

Fig. 3. Block diagram of the optimization technique proposed in this paper.

\section{APPLICATION}

To exemplify the use of the presented technique, let us consider the design of a microstrip magnetic dipole for operation at $f_{0}=2.44 \mathrm{GHz}$ (ISM band). This antenna is printed on a $1.524-\mathrm{mm}$ thick Arlon CuClad 250GX microwave laminate from a lot with $\varepsilon_{r}=2.596$ (after characterization of a laminate sample) and $\tan \delta=0.0018$, and fed through a 50- $\Omega$ SMA coaxial connector having an inner conductor radius $r_{f}=0.65 \mathrm{~mm}$. There are 5 metallized vias placed on each side edge of the patch, 18 metallized 
vias placed on its back edge, $q=4$, and $y_{f}=0$. Moreover, the front edge of the patch lies on the laminate midline along the $y$-direction and the laminate form factor is square with $W=L=125 \mathrm{~mm}$. The full-wave electromagnetic simulations were run using Ansys HFSS $^{\circledR}$ software, being the optimization problem (13) solved using the patternsearch function available in Matlab ${ }^{\circledR}$ [24]. The maximum number of nonzero terms needed for the infinite summations of (4) to converge throughout the design process was 47 and a minimum of 4 converged passes were set in Ansys HFSS ${ }^{\circledR}$. Besides, each full-wave simulation took about $15 \mathrm{~min}$ to run in a computer with Intel ${ }^{\circledR} \mathrm{Core}^{\mathrm{TM}} \mathrm{i} 7$ processor and 16 GB RAM. This computer was also used to run the optimization routine (Step 3) which took less than one minute to converge at every iteration.

After running three iterations of the design procedure described in Section II, the antenna was tuned at the desired frequency of $2.44 \mathrm{GHz}$. Table I summarizes values for patch length $(a)$ and probe position $\left(x_{f}\right)$ along the optimization process and Table II depicts the corresponding correction factors that solve (13). Note that solutions to (13) at each iteration are not unique since the formulation in (13) is not capable of decoupling all corrections factors, e.g., $\Delta a$ and $a_{f}$ which define the equivalent cavity size on $x$-axis. Nevertheless, this does not pose a problem from a physical standpoint as long as the conditions described in Step 3 be met.

Fig. 4 presents a comparison between the input impedance given by (4) (cavity model) and the one obtained in Ansys HFSS ${ }^{\circledR}$ (full-wave simulation) for the three iterations. As seen, the approximated formulas for the correction factors (1st iteration) imply a frequency shift of $37 \mathrm{MHz}$ between the curves from cavity model and full-wave simulation in addition to a difference of approximately $15 \Omega$ in the maximum input resistances. Fig. 5 in turn shows the plots of $\left|\Gamma_{i n}\right|$ versus frequency for the three fullwave simulations. As can be noted, the reflection coefficient level relative to the previous simulation is greater than $-35 \mathrm{~dB}$ at $2.44 \mathrm{GHz}$, as well as the bandwidth for $\left|\Gamma_{i n}\right| \leq-10 \mathrm{~dB}$ is equal to $30 \mathrm{MHz}$.

TABLE I. DIMENSIONS THROUGHOUT DESIGN PROCESS

\begin{tabular}{cccc}
\hline Full-wave simulation & 1st & 2nd & 3rd \\
\hline$a(\mathrm{~mm})$ & 21.20 & 21.52 & 21.51 \\
$x_{f}(\mathrm{~mm})$ & 4.97 & 5.77 & 5.77 \\
\hline
\end{tabular}

TABLE II. CORRECTION FACTORS THROUGHOUT DESIGN PROCESS

\begin{tabular}{cccc}
\hline Parameter & 1st & 2nd & 3rd \\
\hline$\Delta a(\mathrm{~mm})$ & -1.03 & -0.25 & -0.23 \\
$a_{f}(\mathrm{~mm})$ & 0.78 & 1.15 & 1.20 \\
$\Delta b(\mathrm{~mm})$ & -0.91 & -0.96 & -0.83 \\
$w_{f}(\mathrm{~mm})$ & 2.60 & 2.76 & 2.71 \\
$\tan \delta_{e}$ & 0.0190 & 0.0188 & 0.0186 \\
\hline
\end{tabular}

To verify the theoretical data, a prototype was assembled (Fig. 6) and then tested in an anechoic chamber. This prototype was fabricated using a PCB milling machine and its metallized via holes were built with metallic ink. The prototype reflection coefficient is also presented in Fig. 5. As one may observe, there is an excellent match between simulated and experimental results. 
The co-polar patterns obtained using the magnetic current density in (7) and through the full-wave simulation in the third iteration were compared to those ones measured in anechoic chamber. Fig. 7 shows these patterns in both $E$ - and $H$-planes at $2.44 \mathrm{GHz}$. Once again, the performances obtained in full-wave simulation and prototype measurements have very good similarity. It is worth noting that the patterns calculated using the magnetic current density consider an infinite ground plane, consequently they do not exhibit back radiation. This leads to the differences observed between the patterns in green and the others. However, such differences do not significantly impact the effective loss tangent estimate, as it can be seen by comparing the values of $\tan \delta_{e}$ in the first and last iterations (Table II).

The measured cross-polarization level is below $-30 \mathrm{~dB}$ in $E$ - and $H$-planes at $2.44 \mathrm{GHz}$; on account of that, the cross-polar patterns were not depicted. Moreover, the measured gain through gain-transfer method at $2.44 \mathrm{GHz}$ is equal to $(6.1 \pm 1.4) \mathrm{dBi}$ value that is quite close to the $6.5 \mathrm{dBi}$ computed in fullwave simulation.

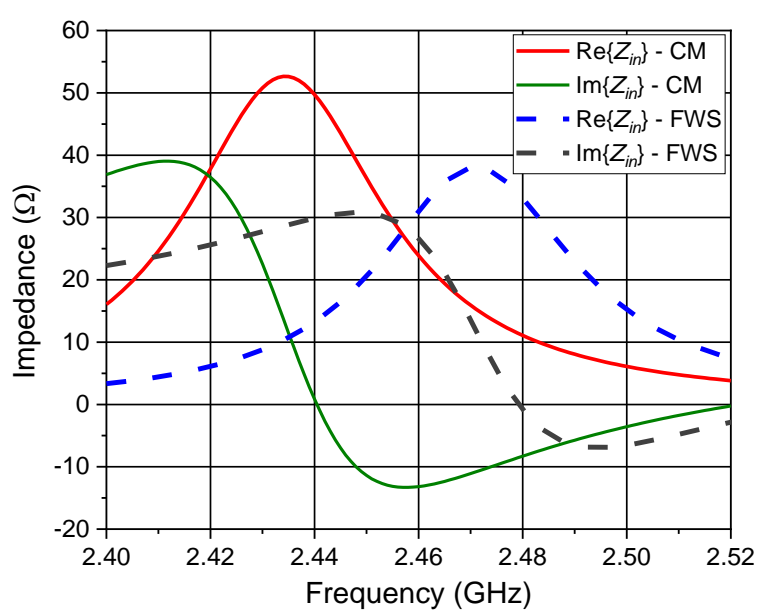

(a) 1 st iteration

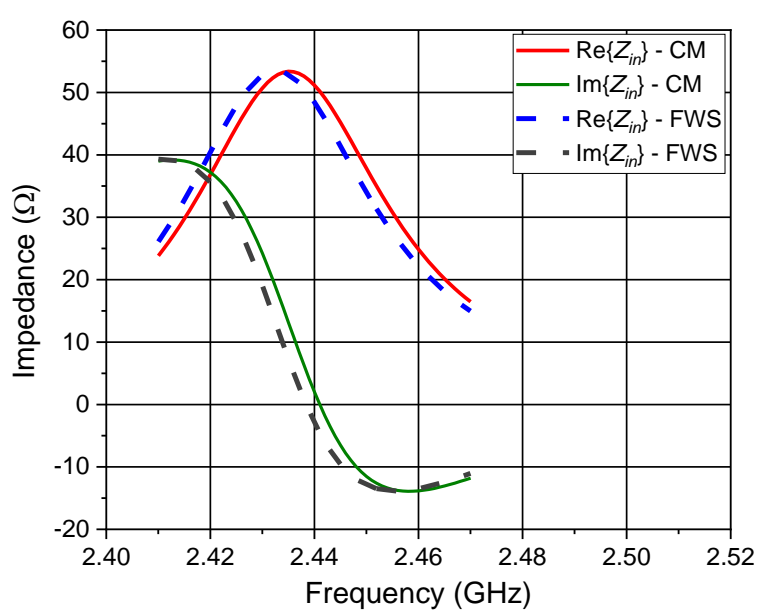

(b) 2nd iteration

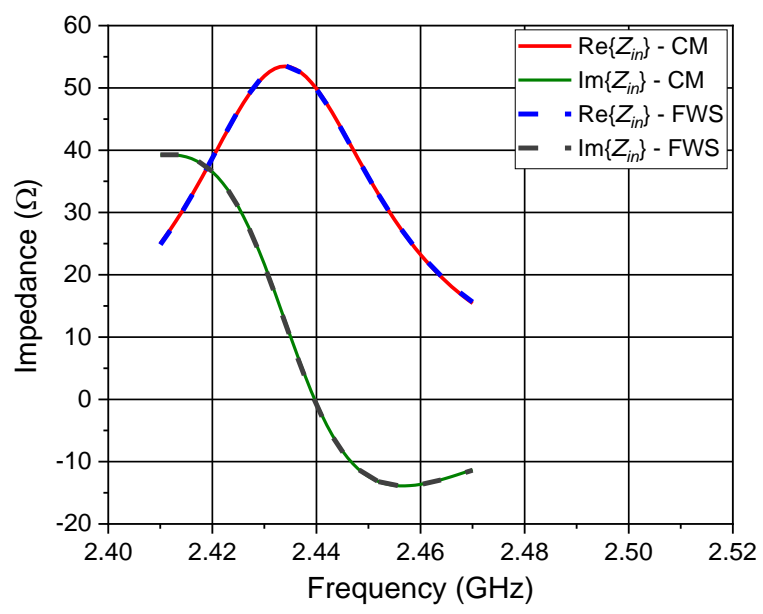

(c) 3rd iteration

Fig. 4. Input impedance along the design process. Cavity Model - CM and Full-Wave Simulation - FWS results. 


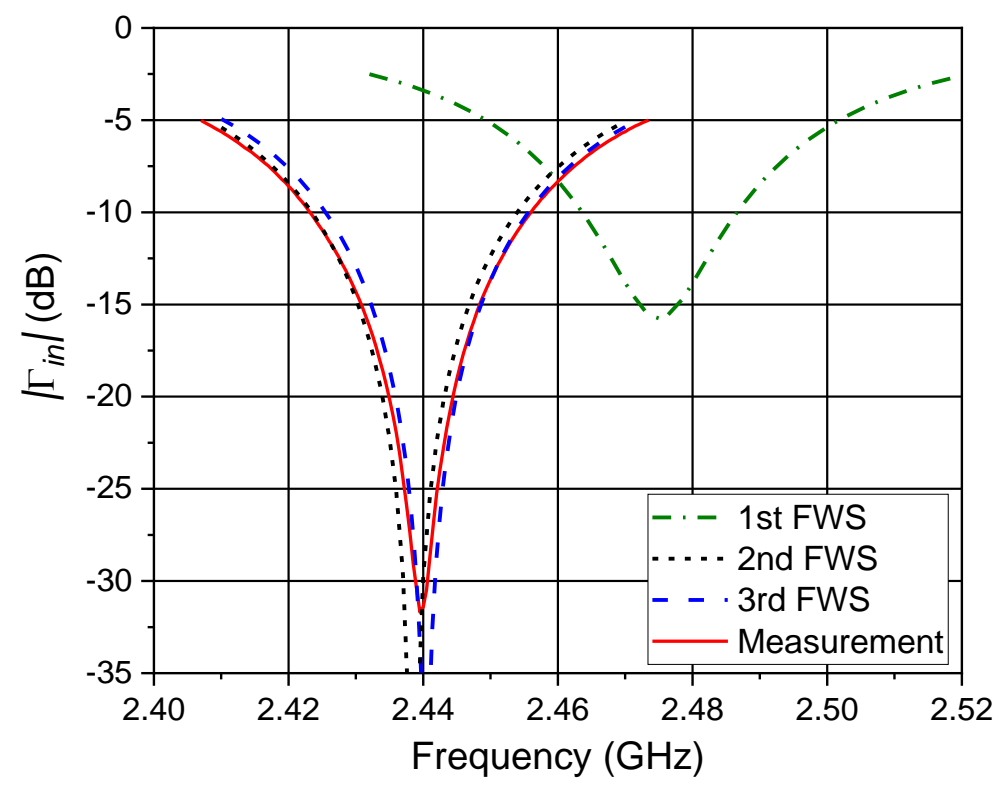

Fig. 5. Reflection coefficient magnitude - microstrip magnetic dipole. Full-Wave Simulation - FWS.

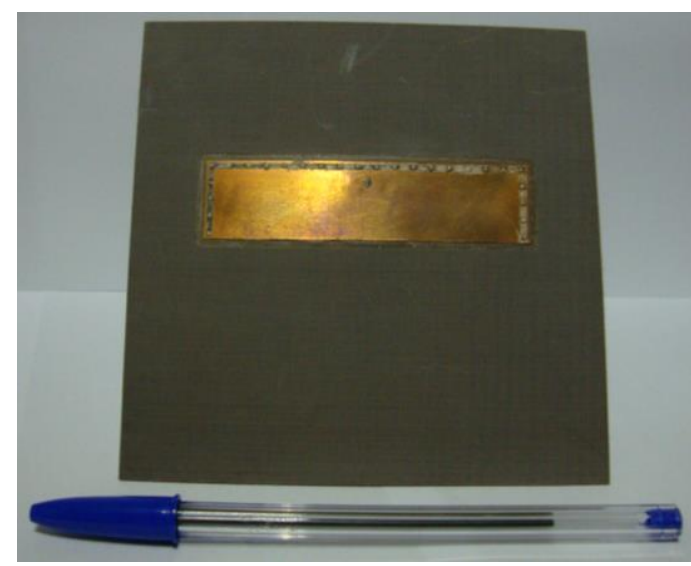

(a)

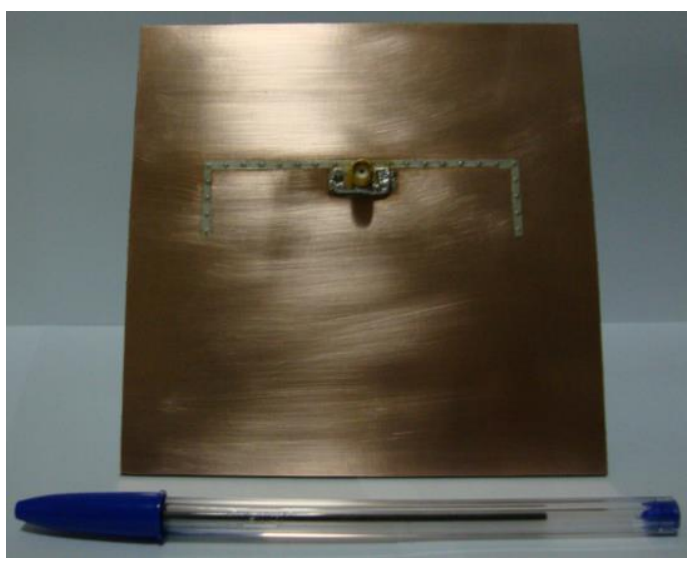

(b)

Fig. 6. Photograph of fabricated microstrip magnetic dipole: (a) Front view; (b) Back view.

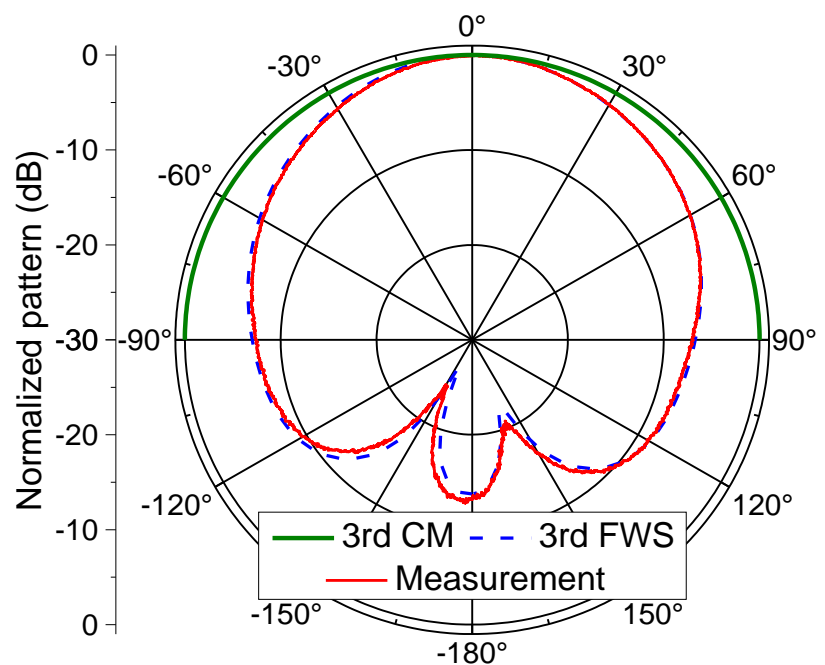

(a)

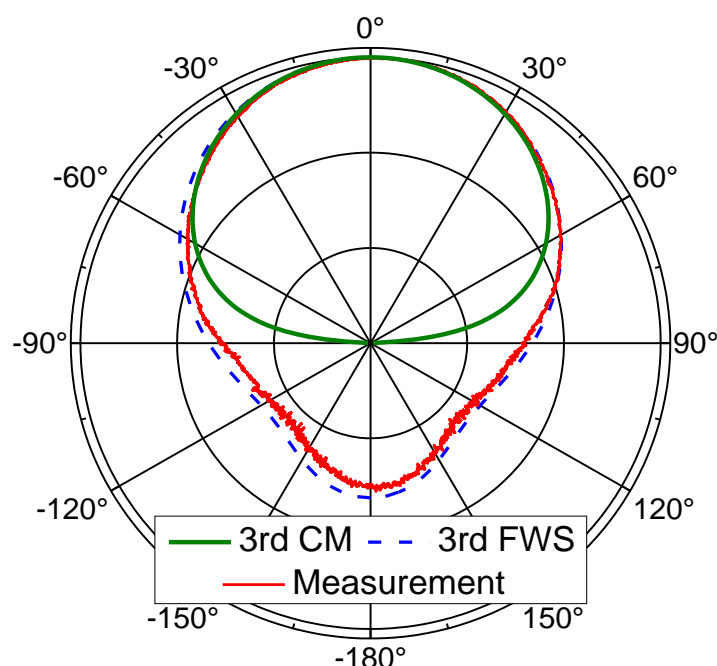

(b)

Fig. 7. Co-polar patterns at $2.44 \mathrm{GHz}$ : (a) E-plane; (b) $H$-plane. Cavity Model - CM and Full-Wave Simulation - FWS.

Brazilian Microwave and Optoelectronics Society-SBMO Brazilian Society of Electromagnetism-SBMag received 11 Oct 2019; for review 17 Oct 2019; accepted 25 Jan 2020 (c) 2020 SBMO/SBMag (cc) BY 


\section{CONCLUSION}

In this paper, an efficient technique able to assist the design of microstrip magnetic dipoles and that explores the physics behind the dipoles behavior is proposed. As observed, it employs a surrogate model that is an extension of the cavity model for a rectangular patch antenna with three metallic vertical walls. The electric field in the equivalent cavity is represented by modal matching that replaces the expansion in resonant modes, due to the proposed method involves only a single summation unlike the second method that requires the evaluation of a double summation. The correction factors inherent to the cavity model, in turn, have been improved throughout the design process based on full-wave simulation data, which enables the tuning of the patch length and probe position in a straightforward manner. The technique is exemplified by a design which is performed with only three full-wave simulations and takes less than one hour to complete. The prototype crosspolarization level revealed itself very low: less than $-30 \mathrm{~dB}$ in both principal planes.

The developed technique can be easily extended to other geometries of patch antennas and printed lines whose analysis models involve correction factors which are not accurately computed through analytical formulas. As stated throughout this paper, parametric analyses could be adopted in most of these designs, since they do not often involve many tuning variables. But in general, parametric analyses themselves do not show the physics behind antenna operation, that is an aspect that may possibly limit the use of some of the antenna capabilities.

\section{REFERENCES}

[1] X.-L. Liang, S.-S. Zhong, and W. Wang, "Design of a dual-polarized microstrip patch antenna with excellent polarization purity," Microw. Opt. Technol. Lett., vol. 44, no. 4, pp. 329-331, Feb. 2005, 10.1002/mop.20625.

[2] J. Yin, Q. Wu, C. Yu, H. Wang, and W. Hong, "Low-sidelobe-level series-fed microstrip antenna array of unequal interelement spacing," IEEE Antennas Wireless Propag. Lett., vol. 16, pp. 1695-1698, 2017, 10.1109/LAWP.2017.2666427.

[3] Z. Yang, L. Zhang, and T. Yang, "A microstrip magnetic dipole Yagi-Uda antenna employing vertical I-shaped resonators as parasitic elements," IEEE Trans. Antennas Propag., vol. 66, no. 8, pp. 3910-3917, Aug. 2018, 10.1109/TAP.2018.2835673.

[4] Y.-Q. Wen, B.-Z. Wang, and X. Ding, "A wide-angle scanning and low sidelobe level microstrip phased array based on genetic algorithm optimization," IEEE Trans. Antennas Propag., vol. 64, no. 2, pp. 805-810, Feb. 2016, 10.1109/TAP.2015.2507173.

[5] T. Li, F.-S. Zhang, F. Gao, Q. Zhang, and Y.-L. Guo, "Microstrip magnetic dipole Yagi antenna with enhanced impedance bandwidth and reduced size for wideband wireless applications," Prog. Electromagn. Res. C, vol. 73, pp. 105-113, 2017, 10.2528/PIERC17021902.

[6] Y.-Q. Wen, B.-Z. Wang, X. Ding, and R. Wang, "Wide-angle scanning phased array based on microstrip magnetic dipole Yagi sub-arrays," in Proc. AP-S/URSI, Vancouver, BC, Canada, 2015, pp. 2493-2494.

[7] L. Yang, J.-D. Zhang, and W. Wu, "Wideband microstrip series-fed magnetic dipole array antenna," Electron. Lett., vol. 50, no. 24, pp. 1793-1795, Nov. 2014, 10.1049/el.2014.3434.

[8] J. Liu and Q. Xue, "Microstrip magnetic dipole Yagi array antenna with endfire radiation and vertical polarization," IEEE Trans. Antennas Propag., vol. 61, no. 3, pp. 1140-1147, Mar. 2013, 10.1109/TAP.2012.2230239.

[9] D. B. Ferreira, "Radiation pattern synthesis of planar and spherical microstrip antenna arrays," D.Sc. thesis, Dept. Microw. Optoelectron., Aeronautics Inst. Technol., Sao Jose dos Campos, Brazil, 2017.

[10] S. Koziel and A. Bekasiewicz, Multi-objective design of antennas using surrogate models. Hackensack, NJ, USA: World Scientific, 2016, pp. 81-85.

[11] C. B. Paula, D. C. Nascimento, and I. Bianchi, "An efficient technique for design of electrically thick differentiallydriven probe-fed microstrip antennas," Prog. Electromagn. Res. M, vol. 40, pp. 37-44, 2014, 10.2528/PIERM14110304.

[12] S. Koziel and S. Ogurtsov, "Simulation-based design of microstrip linear antenna arrays using fast radiation response surrogates," IEEE Antennas Wireless Propag. Lett., vol. 14, pp. 759-762, 2015, 10.1109/LAWP.2014.2377519.

[13] T. Khan and A. De, "Design of circular/triangular patch microstrip antennas using a single neural model," in Proc. 2011 IEEE Appl. Electromagn. Conf. (AEMC), Kolkata, India, 2011, pp. 1-4. 
DOI: http://dx.doi.org/10.1590/2179-10742020v19i11898

[14] J. L. Chávez-Hurtado and J. E. Rayas-Sánchez, "Polynomial-based surrogate modeling of RF and microwave circuits in frequency domain exploiting the multinomial theorem," IEEE Trans. Microw. Theory Techn., vol. 64, no. 12, pp. 4371-4381, Dec. 2016, 10.1109/TMTT.2016.2623902.

[15] E. Penard and J. P. Daniel, "Open and hybrid microstrip antennas," IEE Proc., vol. 131-H, no. 1, pp. 38-44, Feb. 1984, 10.1049/ip-h-1.1984.0008.

[16] R. Garg, P. Bhartia, I. Bahl, and A. Ittipiboon, Microstrip Antenna Design Handbook. Norwood, MA, USA: Artech House, 2001.

[17] K. Wu, D. Deslandes, and Y. Cassivi, "The substrate integrated circuits - a new concept for high-frequency electronics and optoelectronics," in Proc. 6th Int. Conf. Telecommun. Modern Satellite, Cable Broadcast. Service, Nis, Yugoslavia, 2003, pp. P-III-P-X.

[18] W. Wen et al., "Equivalence between substrate-integrated (SIRW) rectangular waveguide short-circuit load and its equivalent rectangular waveguide short-circuit load," Microw. Opt. Technol. Lett., vol. 48, no. 9, pp. 1694-1698, Sep. 2006, 10.1002/mop.21805.

[19] D. Thouroude, M. Himdi, and J. P. Daniel, "CAD-oriented cavity model for rectangular patches," Electron. Lett., vol. 26, no. 13, pp. 842-844, Jun. 1990, 10.1049/el:19900552.

[20] K. F. Lee, K. M. Luk, and H. W. Lai, Microstrip Patch Antennas, 2nd ed. Singapore: World Scientific, 2018.

[21] Y. Lo, D. Solomon, and W. Richards, "Theory and experiment on microstrip antennas," IEEE Trans. Antennas Propag., vol. 27, no. 2, pp. 137-145, Mar. 1979, 10.1109/TAP.1979.1142057.

[22] E. O. Hammerstad, "Equations for microstrip circuit design," in Proc. 5th Eur. Microw. Conf., Hamburg, Germany, 1975, pp. 268-272.

[23] C. A. Balanis, Antenna Theory: Analysis and Design, 4th ed. Hoboken, NJ, USA: Wiley, 2016.

[24] Matlab, MathWorks. Patternsearch (R2019a). (2019) [Online]. Available: https://www.mathworks.com/help/gads /patternsearch.html, Accessed on: Sep. 11, 2019. 